\title{
Bioanalysis
}

\section{Bias in clinical chemistry}

Clinical chemistry uses automated measurement techniques and medical knowledge in the interest of patients and healthy subjects. Automation has reduced repeatability and day-to-day variation considerably. Bias has been reduced to a lesser extent by reference measurement systems. It is vital to minimize clinically important bias, in particular bias within conglomerates of laboratories that measure samples from the same patients. Small and variable bias components will over time show random error properties and conventional random-error based methods for calculating measurement uncertainty can then be applied. The present overview of bias presents the general principles of error and uncertainty concepts, terminology and analysis, and suggests methods to minimize bias and measurement uncertainty in the interest of healthcare.

\section{Background}

Every year clinical chemistry laboratories commonly measure in the order of 20 measurands of samples from an average person. It takes highly automated measurement methods and systems, combined with advanced information technologies to accomplish this mammoth task. Physicians increasingly rely on measurement results for objective diagnosis and monitoring of treatment effects. They can also be used when assessing the overall quality of treatment/healthcare as exemplified by the use of the glycosylated hemoglobin to monitor the overall quality of diabetes treatment.

Measurements in clinical chemistry are performed at several 'levels' of healthcare, from large university hospitals or commercial laboratories to local hospital laboratories or physicians' surgeries to measurements performed by the patients themselves. Minimal criteria for the performance of individual measurement methods and systems are frequently decided by national or international organizations and, consequently, their performance is monitored accordingly. Even if individual measurement methods and measuring systems fulfill each minimal criteria, all possible steps may not have been taken to minimize the overall measurement uncertainty for all available methods that samples from individual patients are likely to encounter over time. Healthcare decisions for individual patients are influenced by the results of all measurements, irrespective of which measurement methods and systems they originate from. Bias between measurement methods and systems, therefore, still represents a substantial challenge in clinical chemistry, in particular for the frequently used immunochemical methods that rely on reagents that vary substantially between producers, for example, those regarding epitope specificities.

Uncertainty of the high-volume measurement methods in clinical chemistry has decreased substantially in recent decades with the advent of highly automated measurement methods and reference measurement systems. The most substantial improvements have been accomplished in reducing the repeatability and day-to-day variation. Bias has also been decreased, but not to the same extent. Bias currently represents a much more formidable challenge than repeatability and day-to-day variation when attempting to further reduce measurement uncertainty (Figure 1).
Elvar Theodorsson*,1, Bertil Magnusson ${ }^{2}$ \& Ivo Leito ${ }^{3}$ 'Department of Clinical Chemistry \& Department of Clinical \& Experimental Medicine, Linköping University, Linköping, Sweden ${ }^{2}$ SP Technical Research Institute of Sweden, Borås, Sweden ${ }^{3}$ Institute of Chemistry, University of Tartu, Estonia

*Author for correspondence:

Tel.: +467 36209471

elvar.theodorsson@liu.se 


\section{Key terms}

Bias: Trueness is the "closeness of agreement between the average of an infinite number of replicate measured quantity values and a reference quantity value". It is quantitatively expressed as bias.

Secondary adjustment: Secondary adjustment (usually by linear regression) of the results from a properly calibrated adept method in order to eliminate its possible bias from the mentor method.

Matrix effects: The combined effect of all components of the sample other than the analyte on the measurement of the measurand. If a specific component can be identified as causing a matrix effect then this is referred to as interference.

There is general agreement on the fundamental role of reference measurement systems in clinical chemistry to this end, but no general agreement has yet been reached on user-conducted secondary adjustment by means of fresh patient samples in order to minimize or eliminate bias. Eliminating the clinically most important bias is an important task for laboratory organizations providing service to patients and healthcare personnel $[2,3]$.

Measurement uncertainty encloses the interval of measurement results within which the true value of the measured quantity lies with some predefined (usually 95\%) probability. Although, strictly speaking, measurement uncertainty is a property of a measurement result and determines what use the measurement results can be put to, it can also be used for characterizing results in general from measurement methods as

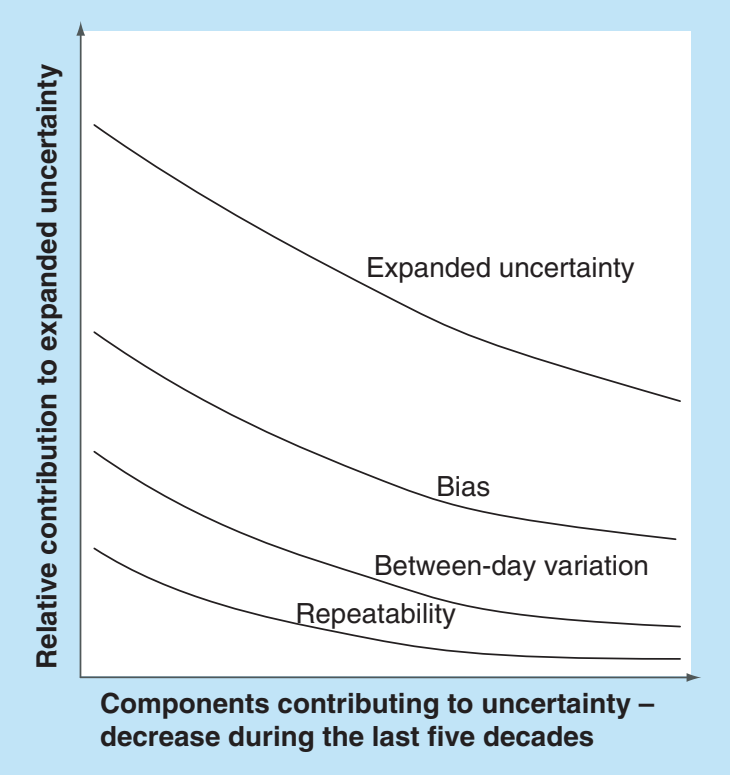

Figure 1. Currently, repeatability and day-to-day variation contribute less than bias to the expanded uncertainty of high-volume measurement methods in clinical chemistry. in uncertainty calculations of the guide to the expression of uncertainty in measurement (GUM) [4], total error [5] or RiLi-BAEK methods [6] or variations of them.

This paper is an overview of: the internationally agreed concepts and calculation methods used for handling bias for a single laboratory with one method, and for a laboratory with several methods and locations; the different ways of investigating the causes of bias; and; practical remedies for minimizing bias in clinical chemistry.

\section{Measurement}

Measurement is the "process of experimentally obtaining one or more quantity values that can reasonably be attributed to a quantity" [7,8]. A quantity can be a concentration and, therefore, a quantity value is the result expressed in concentration. We do not directly measure the molecule of interest but rather rely on a physiochemical property, "kind of quantity" [8], that sufficiently characterizes the molecule for the intended purpose of measurement, for example, absorbance of light at a certain wavelength, elution time from a chromatographic column, immunologic reactivity etc. This is the reason that the term used for what we measure is 'measurand'; it is the "quantity intended to be measured" [8]. The calibrators used are of well-defined origins and have assigned concentrations, which are traceable to internationally accepted standards. The functional relation between assigned concentrations of the measurand in the calibrators on the kind of quantity measured in the samples is established and used to estimate the concentrations in the unknown samples. This means that we run the risk that a multitude of factors other than the concentrations of the molecules intended to measured (confounding factors), including interferences and matrix effects, influence the measurement results, resulting in increased measurement errors (systematic and/or random errors) and measurement uncertainty. Minimizing measurement uncertainty in the interest of patient care is a prerequisite for a well-functioning clinical chemistry laboratory. Therefore, it is of interest to minimize all factors contributing to measurement uncertainty of the measurement results, especially the bias, which in most cases today is the major uncertainty component.

\section{Terminology to describe results of measurement \& their quality}

The following terms are essential when describing and assessing the measurement quality: the general terms measure and, measurement uncertainty, traceability and verification; and the specific terms trueness, precision and accuracy. The definitions and principles 
for use can be found in two internationally agreed documents written by the international organization for standardization (ISO) technical advisory group 4 (TAG4) of The joint committee for guides in metrology (JCGM). international bureau of weights and measures: The international vocabulary of metrology - basic and general concepts and associated terms (VIM); and GUM. The organizations that send their representatives to the JCGM meetings are: the international electrotechnical commission (IEC), the international federation of clinical chemistry and laboratory medicine (IFCC), ISO, the international union of pure and applied chemistry, the international union of pure and applied physics, the international organization of legal metrology and the international laboratory accreditation cooperation.

Important organizations within metrology in the English-speaking countries are absent from this list, for example,, clinical and laboratory standards institute (CLSI) and US FDA. The principles established in the VIM and GUM are as yet not as widely adopted in these countries as in other parts of the world. Furthermore, it still remains difficult to differentiate between colloquial and 'scientific' English in the field of metrology. Examples are the use of the concept of 'accuracy' when meaning 'trueness' and the concept of 'analyte' when meaning 'measurand'. However, important authorities in the English-speaking countries, including the US FDA [9] and CLSI are increasingly adopting the international nomenclature, for example, using accuracy to describe the combination of random and systematic error.

Metrology is an important subject in many fields of knowledge and it is, therefore, crucial for correct understanding and application that individuals in all fields use the concepts and terms that have been internationally discussed and compromised on $[8,10]$, even if it takes practicing the use of new concepts and words.

A qualitative concept measurement trueness is the "closeness of agreement between the average of an infinite number of replicate measured quantity values and a reference quantity value" [8]. It is quantitatively expressed as bias. Another qualitative concept, measurement accuracy, describes the "closeness of agreement between a measured quantity value and a true quantity value of a measurand" [8]. It includes both systematic and random error components.

A more accurate result has a smaller measurement error. It is, on average, more true when the bias is small and more precise when the random error is small Figure 2.

Repeated measurements of the measurand (in the case of chemical measurements it is concentration of an analyte) in the same sample make up a frequency distribution of values, which contains important information on the inherent properties of the measurement method. This frequency distribution is commonly the Gaussian/Normal distribution. If a summary of the frequency distribution by a single number is needed, the center $=$ average $=$ mean $=$ expected value is the most logical choice. The variance and its square root, the standard deviation, describe the distribution of the random variable.

Measurement error or error is a property of a single measurement: "measured quantity value minus a reference quantity value" [8].

When two or more replicate measurements are made, the mean of the obtained values is more likely to be closer to the reference quantity value/true value than a single value since repeated measurements decrease the effect of the random error on the mean (Figure 3). Importantly, repeated measurements do not decrease the effects of the systematic error (bias) on the measurement result. Effects of the random error on the mean are, therefore, improved by increased number of repeated measurements, whereas the systematic error is not influenced.

Measurement bias is "estimate of a systematic measurement error" [8]. Systematic measurement error, in turn, is "component of measurement error that in replicate measurements remains constant or varies in a predictable manner" [8]. Systematic measurement error is the "closeness of agreement between the average of an infinite number of replicate measured quantity values and a reference quantity value" [5]. Commonly in clinical chemistry bias is taken to be the difference between a measured average and a conventional or reference quantity value. However, the measurement conditions need to be stated - laboratory bias should normally be reported based on results measured under intermediate precision conditions (see below).

\section{Repeatability, reproducibility, measurement uncertainty \& expanded measurement uncertainty}

The different component of measurement uncertainty can be illustrated by the laboratory ladder, as originally conceived by Thompson [12]. Repeatability is 'measurement precision under a set of repeatability conditions of measurement' [8]. It is commonly expressed as standard deviation or relative standard deviation/coefficient of variation - step 4 in Figure 4.

Reproducibility condition of measurement is 'condition of measurement, out of a set of conditions that includes different locations, operators, measuring systems, and replicate measurements on the same or similar objects' $[8]$ - all steps in Figure 4B.

Intermediate precision condition of measurement is 'under a set of intermediate precision conditions of 


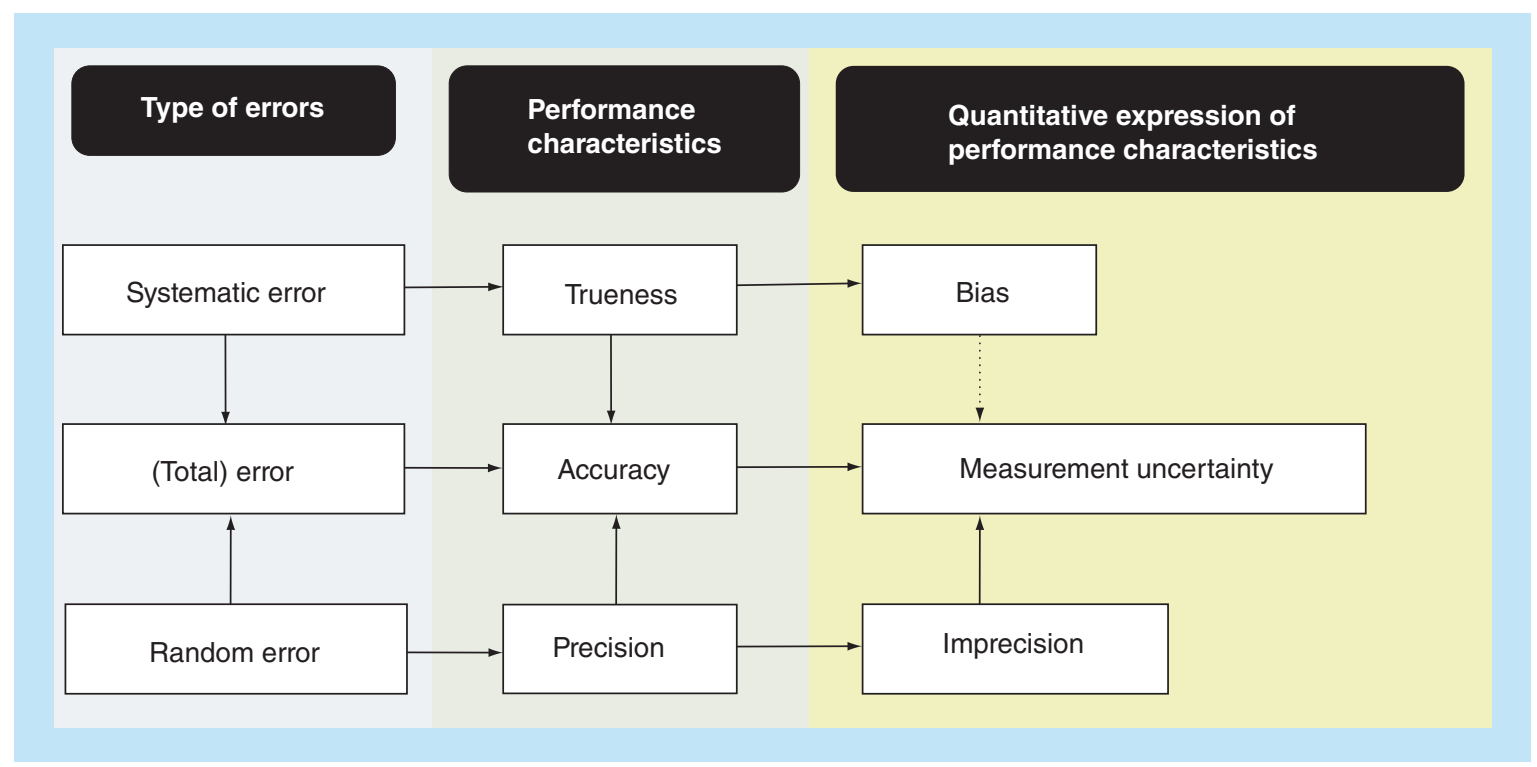

Figure 2. Concept diagram [8]. Explaining the relationships between concepts describing random and systematic errors, as well as measurement uncertainty. The dotted line from bias to measurement uncertainty is to indicate that if bias can be estimated, it should be eliminated.

Adapted with permission from [11] @ Springer (2007).

measurement', out of a set of conditions that includes the same measurement procedure, same location and replicate measurements on the same or similar objects over an extended period of time, but may include other conditions involving changes $[8]-$ steps 3 and 4 in Figure 4A, and all steps in Figure 4B. It is commonly expressed as standard deviation or relative standard deviation. Intermediate precision is also called within-laboratory reproducibility.

Standard uncertainty is 'measurement uncertainty expressed as a standard deviation' [8].

Combined standard uncertainty is standard measurement uncertainty that is obtained by combining the individual standard uncertainties estimated for the result.

Expanded measurement uncertainty is the product of a combined standard uncertainty, $\mathrm{u}_{\mathrm{c}}$, and a coverage factor, $k$. Using a coverage factor of two with sufficient degrees of freedom means that the value reasonably attributable to the measurand is given with $95 \%$ confidence.

VIM is a normative reference in ISO/IEC 17025:2005 and in ISO/IEC 15189:2012. For chemists, Eurachem has written an introductory guide: Terminology in Analytical Measurement - Introduction to VIM 3 [7]. In the subsequent text we will only discuss the terminology relevant to bias.

\section{Standards, reference measurement systems \& organizations}

Standardization of measurement methods and systems is currently based on the ISO standard 17511:2003
[13]. It details how the metrological traceability of values assigned to calibrators and control materials is established. The calibrators are being used to establish trueness and the control materials to verify or verify trueness of measurement methods or systems.

The joint committee for traceability in laboratory medicine (JCTLM) [14] was established in 2002 in response to the implementation of the European community directive $98 / 79 / \mathrm{EC}$ on in vitro medical devices [15]. Its founding organizations are the International Committee of Weights and Measures, the IFCC and the international laboratory accreditation cooperation. The JCTLM publishes a list of higher order reference materials, reference methods and reference laboratories [16]. The important work performed under the auspices of the JCTLM system for minimizing bias by establishing a reference strengthens the metrological foundations of all measurements in clinical chemistry. They are joined in this effort by other corresponding organizations, including the US FDA, national metrological institutes, etc, in other parts of the world. Although far from easy [17], through perseverance we are likely to see a bountiful harvest of the work done by JCTLM [1623], especially as producers of reagents and systems, and organizers of proficiency testing programs increasingly adopt the facilities that JCTLM brings together.

In 2010, the American association of clinical chemistry initiated the international consortium for harmonization of clinical laboratory results, which is a global effort to harmonize test results $[24,25]$. Among the activities of the consortium is the publication of a toolbox of technical procedures to be considered when devel- 
oping a process to achieve harmonization for a measurand [26]. The toolbox sets out lofty and important goals for the harmonization of calibrators, reagents and measurement systems. However, it does not deal with harmonization of pre- and post-analytical factors, which frequently play a very substantial role in the errors encountered in clinical chemistry [22,27]. The international consortium for harmonization of clinical laboratory results has recently broadened its scope to also include pre- and post-analytical factors [25]. The latter are of particular interest in clinical chemistry.

The European federation of clinical chemistry and laboratory medicine, which represents IFCC in Europe, has several groups with special focus on pre- and postanalytical factors. It also collaborates with groups pf clinical experts on test evaluation, interpretation of results, clinical guidelines, etc [28-31].

Current and future efforts in harmonizing measurement results in clinical chemistry are likely to include extensive co-operation between industry, standardization organizations, professional organizations and individual researchers. They also include all aspects of the process from the decision to use the clinical chemistry laboratory in diagnosis through to preparing the patient, taking and transporting the samples, measuring the samples and reporting the results, and interpretation of the results in the clinical context [32].

\section{Bias}

Bias is the difference between the mean of the test results and the reference value

$$
\text { Bias }=\bar{x}-y_{o}
$$

It is commonly expressed as the fraction of the reference value - the relative bias. Different components of measurement uncertainty including biases are obtained depending on the prevailing measurement conditions. This can be illustrated with the different steps of the 'laboratory ladder' (Figure 4) [12,33].

With improved quality of reagents, systems and procedures, the repeatability and day-to-day component of variation are reduced (steps 4 and 3 in Figure 4). Consequently, the laboratory bias and method/measuring system bias (steps 1 and 2) are becoming increasingly important [34]. Therefore, today, bias is quantitatively the most important component of uncertainty (provided that sampling uncertainty is low) for measurement results in clinical chemistry between laboratories, reagents and reagent lots, measurement methods and systems.

The hierarchy/ladder of measurement uncertainties, including bias, lends itself well to statistical analysis using analysis of variance and variance component analysis [35-37] where the most prominent cause(s) of variation can be identified (causes of both random and systematic errors) and used to minimize them whenever possible and practical. This requires the use of stable control material with appropriate matrix properties (commutability) that is used at all levels of

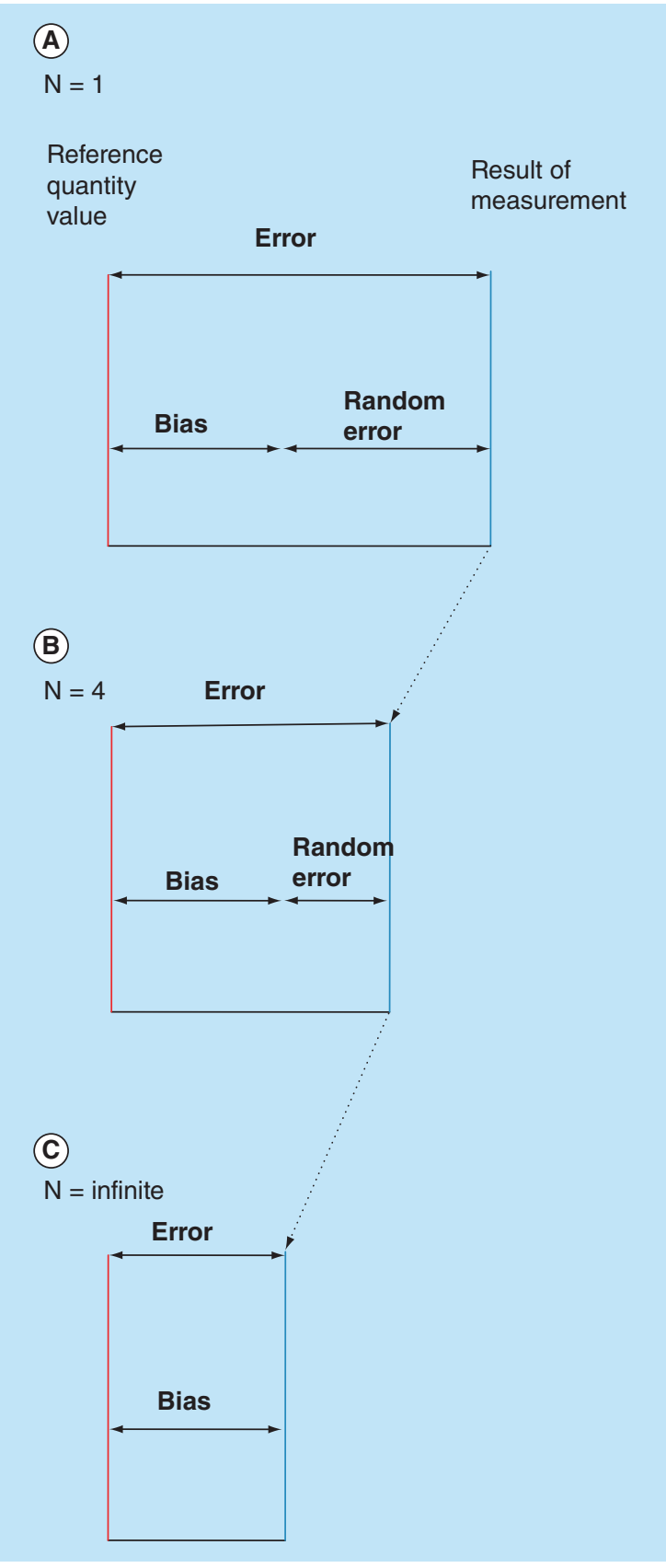

Figure 3. The components of error (random and systematic error) of (A) a single result of measurement, (B) the mean of four replicate measurements and (C) the mean of infinite number of measurements, which eliminates the random error component. The random error component of the uncertainty in determining the mean is inversely related to the square root of the number of observations - the standard error of the mean. 
(A)

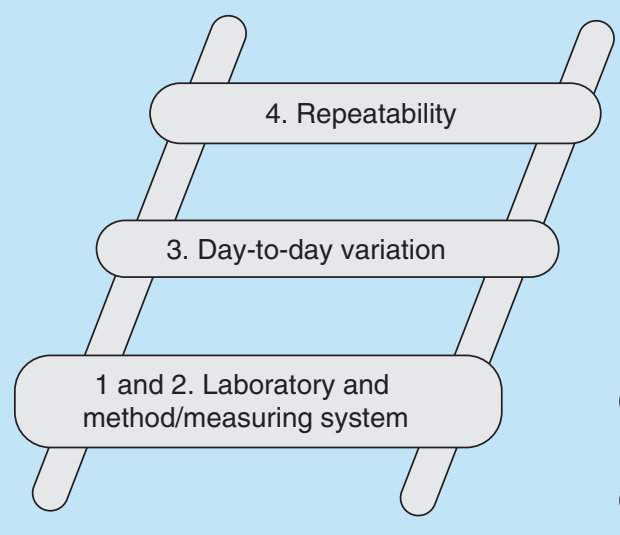

(B)

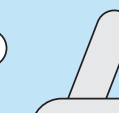

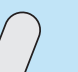
ח

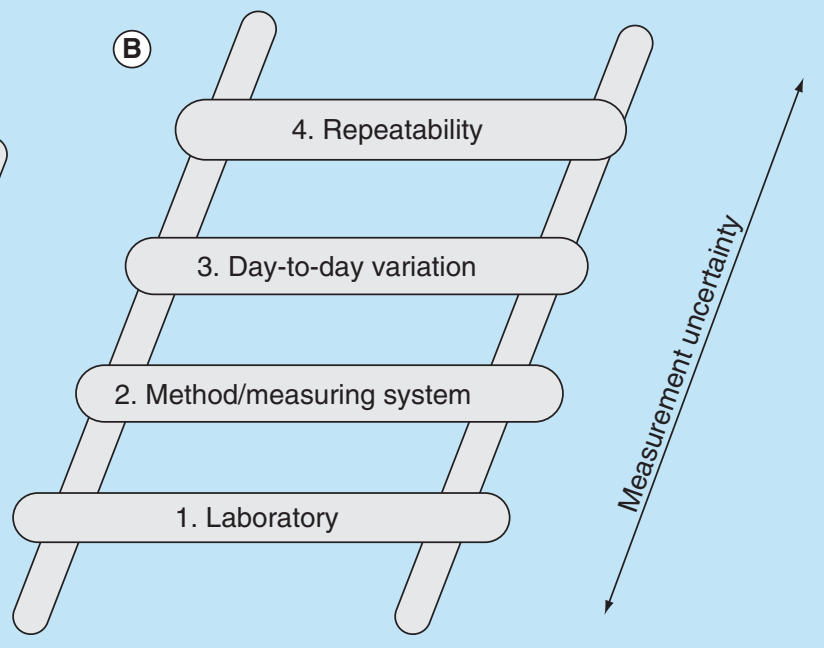

3. Day-to-day variation

Figure 4. The ladder of errors for measures one parameter in clinical chemistry according to the concept

introduced by Thompson et al. [12]. (A) Ladder represents a laboratory using essentially a single measurement method and measurement system for serving their customers. (B) Ladder represents a situation common in clinical chemistry where a laboratory measures samples in a number of different locations, using several methods and measurement systems. Step 1: The laboratory bias - a bias for an individual laboratory. The 'laboratory' can be a single laboratory or a laboratory organization, for exmaple, all laboratories within a community that a patient is using. Step 2: The method and/or measurement system bias. Step 3: The day-to-day variation - a combination of random error and short-term bias owing to, among other factors, time effects, change of reagents and so on. Step 4: The repeatability - the random error occurring between replicate determinations performed within a short period of time.

measurement including the use of patient samples for quality control.

Given the situation where patient samples for measurement of a particular measurand are always measured within the same conglomerate of laboratories, the most important bias to eliminate is bias between the conglomerate laboratories.

\section{Causes of bias}

The reasons for bias in clinical chemistry are numerous, varying in importance between measurement methods, for example:

- Bias when taking samples, for example, samples are sometimes taken when the patient has been walking around and sometimes when he/she has been lying down. When the regulatory systems of the body adapt to gravity, the blood plasma volume is reduced in the order of $10 \%$ from a lying to a standing position, thus increasing the concentration of macromolecules and cells in the blood of the patient;

- Instability of the sample during transport or storage, for example, during transport in extremes of heat and cold, and mechanical effects on cells and blood gases when transporting samples through pneumatic tubes in hospital transport systems;
- Uncorrected loss of measurand at extraction, for example, when preparing samples for measurement using high-performance liquid chromatography or mass-spectrometry;

- Errors when the calibrator is prepared, including errors in volume measurements or in weighing of calibrators in the laboratory;

- Using sample matrix that differs from the matrix in the samples, for example, using de-fatted and lyophilized stable materials for internal quality control or proficiency testing programs;

- Interferences in the samples, for example, the color of hemoglobin and bilirubin in hemolytic and icteric samples, or the presence of high concentrations of proteins or lipids in the sample (myeloma or hyperlipidemia);

- The presence of molecules that specifically interfere with the reagents used in the measurement process, for example, heterophilic antibodies (for example, human antibodies against mouse $\mathrm{IgG}$ );

- Specificity for different epitopes in macromolecules of antibodies used in immunochemical measurement methods, for example, when measuring mac- 
romolecules including prostate-specific antigen, troponins and protein- or peptide hormones.

\section{Clinically important or clinically unimportant bias}

In clinical chemistry the decisions based on measured concentrations of components are used primarily for two purposes: for diagnosis; and for monitoring of treatment results.

When used for diagnosis, the clinical decisions depend on comparison of the central tendency and variation of the concentrations of the component in the population of the subjects used for establishing the reference interval with the central tendency and variation of the concentrations in the population afflicted by the disease [38]. Studies of the properties of diagnostic methods are usually performed using methods of receiver operating characteristics $[39,40]$. The clinical decision on whether a concentration of a measurand in a patient sample belongs to the population of the healthy or to the population of the diseased is influenced by the uncertainty of the measurement result. This uncertainty consists of measurement uncertainty (bias and imprecision), on uncertainty in the sampling and sample handling and on the spontaneous biological variation [41] of the component in the healthy subjects and patients as their homeostatic systems and the possible disease processes influence the concentrations of the measurands in parts of the body where the samples are taken from (usually components of blood, urine or cerebrospinal fluid) [42]. A clinically important bias is a bias which is likely (with a predefined probability - commonly $\mathrm{p}>0.05$ ) to influence the clinical decision between health and disease when studied in the context of all the other uncertainty components involved, including biological variation. A clinically unimportant bias is a bias that does not fulfill this criterion.

The data on biological variation for Hemoglobin A1C and Alanine aminotransferase (Table 1) may be used to illustrate this.

Since the between-individuals biological variation of HbA1C $(5.7 \%)$ is much smaller than for ALAT

\section{Key term}

Commutability: To what extent reference materials, calibrators and control materials show matrix properties similar to those of fresh natural samples. Fresh natural patient samples represent the ultimately commutable materials for comparing measurement methods in clinical chemistry.

(41.6\%), a possible bias in the measurement of the concentrations of $\mathrm{HbA1C}$ is much more likely to influence clinical decisions in diagnosing diabetes mellitus than a possible bias in the measurement of ALAT when diagnosing liver conditions due to the fact that the large (41.6\%) biological variation of ALAT is likely to be the major uncertainty component when the concentrations/activity of ALAT is used for diagnosis. Therefore, a bias of $2 \%$, for example, when measuring the concentrations/activity of ALAT is usually clinically unimportant.

When used for monitoring treatment results within a single patient, the within-individual biological variation determines the uncertainty caused by biological variation. In this instance, sampling and sample handling variation are commonly regarded as constant. When several measurement systems are used for monitoring the patient (for example, self-monitoring instrument, local physician instrument, local hospital instrument, university hospital instruments) bias between the measurement systems becomes crucial [44,45] for two reasons: a bias of $2 \%$, for example, is of similar magnitude and importance as the intra-individual biological variation (1.9\%) and is, therefore, important in the overall uncertainty of the clinical decision; and an increase of $2 \%$ in the concentration/fraction of $\mathrm{HbAlC}$ is known to constitute an increased risk for the patient.

Whether a bias between measurement systems for a certain component is clinically important or unimportant is therefore a question of: knowledge about the medical risk that a certain concentration or change in concentrations implies; whether the measurement is used for diagnosis or for monitoring of the effects of treatment; and knowledge about the biological variation of the component.

\section{Table 1. The within-and-between individual components of biological variation of hemoglobin A1C} and of alanine aminotransferase.

\begin{tabular}{|llll|}
\hline System & Component & $\begin{array}{l}\text { Within-individual biological } \\
\text { variation }(\% \mathrm{CV})\end{array}$ & $\begin{array}{l}\text { Between-individual biological } \\
\text { variation }(\% \mathrm{CV})\end{array}$ \\
\hline Blood & Hemoglobin A1C (HbA1C) & $1.9 \%$ & $5.7 \%$ \\
\hline Serum & $\begin{array}{l}\text { Alanine aminotransferase } \\
\text { (ALAT) }\end{array}$ & $19.4 \%$ & $41.6 \%$ \\
\hline Data taken from [43]. & & \\
\hline
\end{tabular}




\section{Key terms}

Mentor measurement system: A mentor measurement system in a conglomerate of laboratories is taken to be devoid of bias

Variable bias components become random errors over time

It is important to distinguish between short-term (within day) bias and long-term (several months) bias. Many effects causing within-day bias become random effects in the long term. An example is a calibration graph re-made every day (which is quite common in instrumental analysis). Within a given day the small deviations of the calibration graph from an 'ideal calibration graph' affect all the samples in a systematic way. If such small deviations are different on different days then their effect becomes random in the long term. Therefore, during extended periods (weeks and months) of observations many bias components vary and thus increasingly contribute to the random error component (intermediate precision) of the measurement uncertainty. This explains why intermediate precision standard deviation is larger than repeatability standard deviation (if determined correctly): there are many effects that within a given day cause bias and are thus not taken into account by repeatability, but in the long term become random deviations and are thus incorporated into intermediate precision. This is illustrated in Figure 5.

Observation periods lasting for months and years are common in healthcare. Provided that clinically important and large bias components are reduced or eliminated, small bias components, for example those caused by changes in reagent lots and re-calibration of measurement methods, will behave as random errors and routine methods for calculating measurement uncertainty based on random components can be used. In the following discussion of bias, the bias does not refer to the within-day bias but to long-term bias.

\section{Estimation of bias}

The availability of a suitable reference materials is crucial when embarking on the direct estimation of bias. It is mandatory that the material has the following properties: a concentration of the measurand known with sufficiently low uncertainty; it covers the clinically relevant concentration range; and it has an appropriate matrix for the method to be tested. The most common options are: certified reference materials; natural patient samples, for example, plasma, serum or urine, measured using a reference method; or natural samples spiked with a known concentration of the analyte. Note that the reference material used to assess bias must be completely independent from the material used for calibration of the instrument/method - see NOTE 6, 5.13 reference material in VIM [8].

Measurement bias can be estimated using one or more of the following principles:

- Comparing the concentration found by a laboratory's own methods with the stated concentration of a suitable certified reference material;

- Comparing the concentrations obtained by a laboratory's own method in natural samples with the concentrations measured by a reference method in the same sample;

- Participating in proficiency testing schemes. The majority of these programs use consensus concentrations in modified control samples, but some use comparison with reference methods. Evidently the latter are preferable;

- Measuring the recovery of the measurand in spiked natural samples.

In addition, separate investigation of possible bias can be performed:

- By comparing the serial dilution of a natural sample or that of a spiked natural sample with the serial dilution of the calibration curve;

- Studying possible interferences, i.e. selectivity. Selectivity varies among different measurement methods and fields of study. In clinical chemistry the interferences by bilirubin, hemoglobin, lipids, proteins and drugs occur most frequently. Selectivity is "property of a measuring system, used with a specified measurement procedure, whereby it provides measured quantity values for one or more measurands such that the values of each measurand are independent of other measurands or other quantities in the phenomenon, body, or substance being investigated" [5].

If a certified reference material, not used in calibration, with optimal matrix properties is available, it is the best choice for estimating bias. Such materials are produced by recognized authorities according to high standards and is provided together with a certificate stating the reference value of the measurand $\left(y_{o}\right)$ and the uncertainty associated with the determination of the reference value. Certified reference materials in matrix appropriate for all relevant measurement methods are seldom available. Natural patient samples, preferably fresh and available in at least in two clinically relevant concentrations determined by a reference method or a mentor masurement system are therefore frequently used in practice. A reference method or a ref- 
erence measurement procedure is "procedure accepted as providing measurement results fit for their intended use in assessing measurement trueness of measured quantity values obtained from other measurement procedures for quantities of the same kind, in calibration, or in characterizing reference materials" [8].

Due to cost or technical restraints, reference methods are only available for a few, if any, measurands even in university laboratories or in large commercial laboratories. Instead, the laboratories choose a method and a system as an internal reference, a mentor method [46], that can be used, for example, to measure bias between measurement methods and systems within the auspices of the laboratory. It is crucial that the persons responsible for the mentor methods have the appropriate knowledge, skills and interest in maintaining high quality, and interest in eliminating clinically important bias for all measurement methods and systems in their care. Optimal calibrators and stable materials for internal quality control materials should be used, the latter available in quantities for at least 1 year, preferably 2 years, of use. The mentor method should preferably participate in two proficiency testing programs, one based on consensus values and the other based on reference method values, if available. Establishing and maintaining mentor methods in a laboratory organization enables the laboratory organization to estimate and minimize bias for all measurement methods for the same measurand using natural patient samples and, therefore, in the relevant sample matrix (see below).

The bias is calculated for each reference sample as the mean of the test results, $\bar{x}$ minus the reference value; $y_{o}$; Bias $=\bar{x}-y_{o}$.

A positive bias implies that, on average, reported results are too high. Bias is frequently expressed as the fraction of the reference concentration - the relative bias:

$$
\operatorname{Bias}(\text { relative })=\frac{\bar{x}-y_{o}}{y_{o}}
$$

or as percentage:

$$
\operatorname{Bias}(\%)=\frac{\bar{x}-y_{o}}{y_{o}} \times 100
$$

Two different kinds of bias are recognized: constant and proportional to the quantity value. Both can be assumed constant in a narrow concentration interval - the former expressed in an absolute values and the latter expressed in a relative values.

\section{Statistical evaluation of bias when certified} reference materials or materials from reference methods/mentor methods are used

Bias data obtained from internal or proficiency testing programs should not be used for calibration or secondary adjustment. However, they are essential for point-

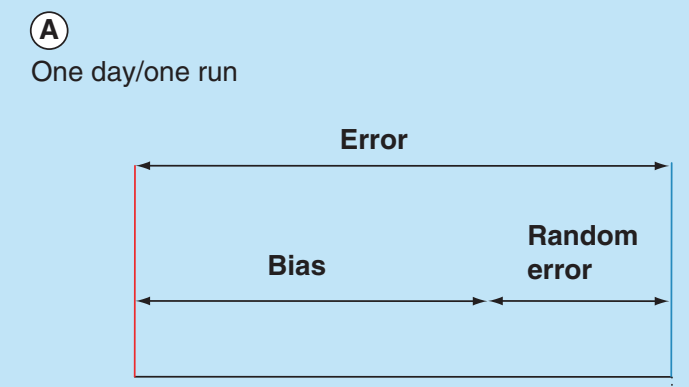

(B)

One week/reagent lot/

calibration

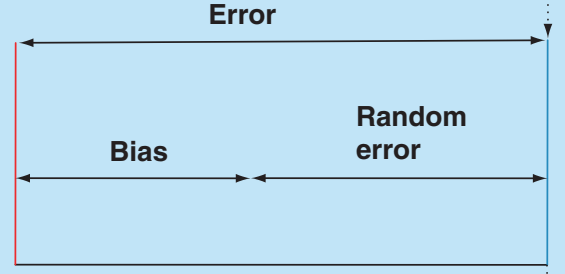

(C)

One year

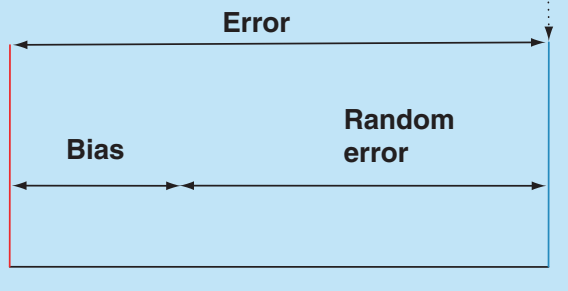

Figure 5. As the length of time observed increases, the random error increases and bias decreases. This is because some bias components become random over time.

ing out which methods or measurement systems are in need of calibration or secondary adjustment.

When determining which methods are in need of secondary adjustment, medical relevance has precedence over statistical significance. Minimizing bias for the concentrations of glycated hemoglobin and free calcium ions has a much larger clinical significance compared with the activity of transaminases in medical decision making.

The measuring method and system to be evaluated should have completed an appropriate process of validation or verification [46] and be in a stable state as judged by the results of internal quality control results [47-49].

It is seldom practicable to detect a bias smaller than the standard deviation [50]. It is also impossible to detect a bias smaller than the uncertainty of the certified reference material used [50]. Information about the uncertainty of the certified reference material is a prerequisite 


\section{Key term}

Mentor-adept method: A method for the systematic use of split samples for secondary adjustment, calibration or quality control. Measurement results from the same sample can either be used for secondary adjustment or for calibration, never for both.

for: determining the relation between the number of replicates needed and the minimum detectable bias; and test for bias and estimating its confidence interval [51].

It is important to make a sufficient number of replicate measurements in order to have adequate power to detect a bias important for the use of the method [50]. The calculations described below suppose that the power of the test should be 0.95 :

$$
\Delta_{D}=\frac{\left(t_{1-\frac{0.95}{2}}+t_{0.95}\right) * s}{\sqrt{n}}
$$

where $\Delta_{D}=$ the bias to be detected; $s=$ the measurement standard deviation; $n=$ the number of replicate measurements; $t=$ Students t-value

An iterative solution is required to calculate the number of observations when the standard deviation and bias are known since the $\mathrm{t}$-value is dependent on the number of observations.

For large number of replicate observations and known standard deviation (for example, from measurement of stable control material), the t-value approaches $\mathrm{z}$, the standard normal deviate [52].

$$
\begin{gathered}
\Delta_{D}=\frac{(1.645+1.960) * \sigma}{\sqrt{n}}=\frac{(3.605) * \sigma}{\sqrt{n}} \\
n \geq 3.605^{2} *\left(\frac{\sigma}{\Delta_{D}}\right)^{2}=13 *\left(\frac{\sigma}{\Delta_{D}}\right)^{2}=\frac{13}{\left(\frac{\Delta_{D}}{\sigma}\right)^{2}}
\end{gathered}
$$

Taking additional factors into consideration shows that 16 replicate measurements are needed to detect a bias equal to one standard deviation (Table 2).

Considerably more statistically advanced methods are available for the estimation of bias and bias uncertainty [53,54]. Practitioners of clinical chemistry have only applied these methods to a limited extent in favor of methods requiring less mathematics/statistics and a more hands-on practical approach.
To correct for bias or not, and by whom?

Magnusson and Ellison [55] have emphasized that situations are common where bias is known but specific corrections cannot be justified. They describe methods for the proper treatment of uncorrected bias and the calculation of its uncertainty [55].

Figure 6 presents the important aspects for deciding whether or not to correct for an observed bias [55,56]. If correction for bias is justified then it must be decided whether the correction should be multiplicative or additive, i.e. if the magnitude of the bias is constant or changes with the concentration level.

There is no point in trying to eliminate or correct a small and clinically unimportant bias, since both elimination and correction need resources and may increase the measurement uncertainty. A small bias that is not eliminated should be included in the calculation of measurement uncertainty as random error. There are no clear guidance on how take into account an observed uncorrected bias as an uncertainty component in the uncertainty estimation. Several options are discussed and modeled in [55].

If the bias is significant and eliminating bias is either impossible or impractical, correcting for bias should be considered. There are three possibilities:

- Correction may be required. If so, correct;

- Correction can be forbidden. If so, do not correct and take the bias into account as an uncertainty component;

- Correction may be allowed. Then we will look at the four further criteria to determine whether correction is justified:

If due to matrix effects, it is amenable to secondary adjustment using mentor-adept methods;

If the cause of bias is not known, correcting is not recommended and it is more reasonable to include bias in the measurement uncertainty estimate. This is because if the cause of bias is not known, in future results the bias may be absent and if we then correct it, the bias may have in fact been increased;

If bias cannot be reliably determined, do not correct for it because if the result is corrected with an unreli-

Table 2. The number ( $n$ ) of replicate observations needed to detect a bias $\Delta_{D}$ expressed in the number of standard deviations $\Delta_{\mathrm{D}} / \sigma$ when the uncertainty in the reference materials can be

\begin{tabular}{|c|c|c|c|c|c|c|c|c|c|c|}
\hline$\Delta_{D} / \sigma$ & 0.5 & 0.6 & 0.7 & 0.8 & 0.9 & 1.0 & 1.5 & 2.0 & 2.5 & 3.0 \\
\hline$n$ & 55 & 39 & 29 & 23 & 19 & 16 & 9 & 6 & 5 & 4 \\
\hline
\end{tabular}
disregarded. To detect a bias of one standard deviation 16 replicate measurements are needed when a confidence limit of 0.95 is required. 
able bias estimate we can make it less accurate than it would have been without the correction;

Correcting for bias is only meaningful if useful reduction of measurement uncertainty is achieved (considering that correcting, while removing bias, also introduces additional uncertainty). If useful uncertainty reduction is not achieved then bias correction is not justified.

In clinical chemistry a medically important bias can and should be eliminated by modifying the method

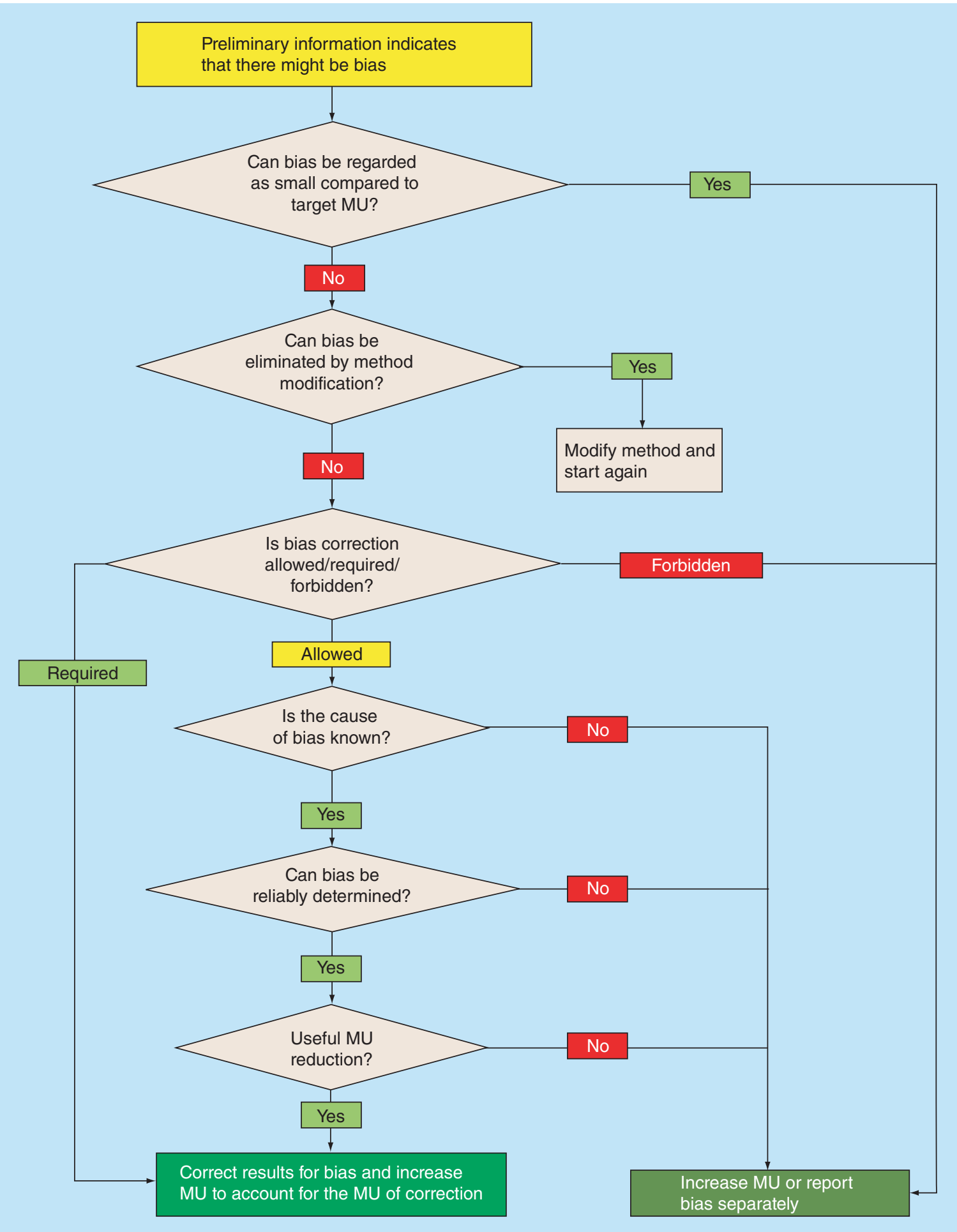

Figure 6. Decision tree when considering whether to eliminate bias [56]. MU: Measurement uncertainty. 
by secondary adjustment. By following this logic, we can frequently answer yes to all of the questions above.

\section{Method bias}

Method bias describes the common situation, particularly for immunoassays, where different epitopes (parts of the molecules intended to be measured) react with the antibodies used. Where macromolecules are involved, in particular where the epitopes of most substantial clinical interest have not been determined or agreed on, antisera from different producers commonly react with different epitopes. In these cases it is usually difficult and commonly impossible to design measurement methods and systems to measure the same concentrations in fresh patient samples, even if the best possible methods of primary calibration are used [26].

A particular challenge is that measurands including follitropin, lutropin, human chorionic gonadotropin and troponin I are present in different molecular forms in different clinical conditions.

\section{Interferences and matrix effects in the samples}

The sample matrix represents 'the components of the sample other than the analyte' [1], and the matrix effect is "the combined effect of all components of the sample other than the analyte on the measurement of the measurand" [1]. The definition adds that "if a specific component can be identified as causing an effect then this is referred to as interference" [1].

Laboratories usually have practical routines for minimizing error caused by taking the sample, transporting it and adding anticoagulants, enzyme inhibitors etc. Producers of measurement methods add substances that minimize the interference of hormone-binding proteins, autoanalyte antibodies and heterophilic antibodies. However, matrix effects may vary in samples from different patients and in particular in processed (for example, de-fatted and lyophilized) control samples, which through matrix effects result in different concentrations measured by different chemical and physiochemical measurement methods.

When appropriate reference measurement system calibrators and methods have been widely applied, different measurement principles and matrix effects constitute the major causes of bias between measurement methods [57-59] (Figure 1). The introduction of enzymatic methodologies have substantially improved the measurement of creatinine, however, at a substantially higher cost than a simpler direct chemical method. The most substantial current obstacles, however, remain in the field of immunochemistry where the producers use antibodies specific for different epitopes of the macromolecules being measured. Even when the best internationally acknowledged calibrators are used for calibration, the concentrations of measurands may differ substantially in patient samples measured with different methods.

The importance of matrix effects in calibration and quality control are especially evident in proficiency testing programs where the same control materials frequently result in substantial differences in mean values between measurement methods and systems urging the companies to create different method-and system groups when reporting the data. Fortunately the bias between the measurement methods is commonly considerably smaller when a freshly taken natural patient sample is measured. Among the reasons for this is that the producers of the measurement methods and systems commonly use natural patient samples when calibrating their methods in relation to reference methods. This underscores the importance of using the most commutable (see below) materials when comparing measurement methods and systems, in particular in proficiency testing programs.

\section{Commutability}

Commutability is a qualitative concept describing to what extent reference materials/calibrators and control materials show matrix properties similar to those of fresh natural patient samples. Fresh natural patient samples, therefore, represent the ultimately commutable materials for comparing measurement methods $[24,25,60-66]$. Natural patient samples are widely used in the industry to ensure that commercially available measurement methods measure the same concentrations in natural patient samples as reference methods, thereby making sure there is an unbroken traceability chain from reference materials to the routinely used measurement procedures $[16,21,62,63,65-67]$.

Results from measurements in patients' samples need to be unbiased by the measurement methods, systems, location and time of testing [63]. TThe most important factor in obtaining this goal is the general use of a reference measuring system by which the result can be traced to a calibrator at a high metrological level [61].

Commutability is also a highly desirable property of stable control materials used for internal quality control and proficiency testing programs during extended time periods, preferably 1-2 years. Commutability and stability are unfortunately opposite properties in this context since lipids are commonly removed and lyophilization frequently used for stabilizing the control materials, thereby substantially changing the matrix. Changed reference materials can, however, be commutable provided the factors changed do not constitute an influence or interference factor. 
The large number of patient samples processed every day in laboratories provide a steady and unique supply of materials with optimal commutability properties for estimating measurement error at no cost. These materials are excellent when used for split-sample/ mentor-adept schemes. The end users of the measurement methods and systems are those who are in possession of this invaluable asset and are able to compare the measurement methods and systems from different producers. The end users are, therefore, in the position to complement the efforts of the different producers, ensuring proper traceability through commutable materials of the reference measurement system all the way down to the measurement of a measurand in a patient sample.

\section{Split-sample/mentor-adept methods for bias estimation \& elimination}

Fresh natural patient samples by definition constitute the sample materials with optimal commutability. This is because measurands measured by different measurement methods and systems should have the same results in the same patient samples given the matrix effects found in natural patient samples [68].

Patient samples must be fresh, properly stored and transported in order to maintain commutability. Using dedicated temperature-controlled transport and measurement within the same day is optimal, but not always practicable. Transport through ordinary mail with varying time in transport, especially in climates with variable temperatures, run the risk of making natural patient samples inferior to stabilized control materials [69,70].

Interferences caused by hemolysis, hyperlipidemia, icterus and hyperproteinemia is usually evident and information about intake of drugs interfering in the measurements is usually available. The presence of matrix effects resulting in different concentrations using different measurement methods and systems is harder, but not impossible, to deal with. A mentor laboratory is appointed amongst laboratories sharing information technologies and leadership. It has particularly well controlled (participation in two separate proficiency schemes) methods, and well educated and dedicated personnel responsible for calibration and quality control. After measurement in any of the other laboratories in the conglomerate, the sample is sent to the mentor laboratory (split-sample technique) for analysis in order to measure the difference. In the long term, the mean difference at a certain concentration is the bias [46]. Split-sample/mentor-adept methods in clinical chemistry are used for secondary adjustment [71] and/or for internal quality control, i.e. for long- time control that the results of the calibration are maintained [46] (Figure 6).

\section{Key term}

Split-sample: A fresh natural sample measured using two measurement systems for the purpose of comparison, calibration or QC.

Is secondary adjustment necessary if reference measuring systems work properly?

Theoretically, the answer is no. The use of calibrators of the highest metrological quality with optimal matrix properties aided by commutable materials including fresh patient samples should solve all outstanding issues. However, in practice, bias is common between measurement methods and systems from the same or different manufacturers. This is an evidence that reference measuring systems as yet do not fulfill all requirements.

If bias is likely to influence clinical decisions, for example, in the case of glycated hemoglobin, thyreotropin, prostate-specific antigen and ionized calcium, exchange of measurement methods or systems showing the most substantial bias may be considered, if economically feasible, otherwise secondary adjustment should be considered.

\section{Secondary adjustment using fresh patient samples sent out}

In order of 20-40 fresh natural patient samples covering a clinically relevant interval of concentrations are sent from a central laboratory (mentor) to the measurement methods and systems subject to secondary adjustment (adept). When the samples have been measured by the adept-measurement method/system, the linear relation between the results of the mentor and the adept are fitted using orthogonal regression methods. This regression equation is then used, preferably in a dedicated computer interface for recalculating the concentrations measured by the adept, resulting in concentrations devoid of bias in relation to the mentor.

Alternatively, two samples pooled from several patients, spanning a clinically relevant concentration interval and both in sufficient volumes to permit at least 15 replicate measurements are used. The mean of at least six replicates $[3,72]$ can be determined a minute contribution from the random error. The equation of this straight line, using two points, can be used for secondary adjustment as described above.

In clinical chemistry, there is need for end user performed elimination of bias for measurement methods, especially when used for diagnosing and monitoring the effects of treatment using target limits for measurement uncertainty which are otherwise difficult to fulfil, for example, in diabetes, hyperlipidaemia and endocrinology $[46,59]$. 
There are guidelines for method comparison using patient samples, for example, CLSI EP09-A3 [2] and recommendation from the highest level of metrology (GUM) [4] to eliminate bias. However, no authoritative guidelines on secondary adjustment have appeared yet, possibly because they may be considered difficult to reconcile with directives and regulations, for example, the EU in vitro directive [15].

\section{Internal quality control using fresh patient samples sent in}

In this situation the mentor sends samples that have already been measured by the method/measuring system to be controlled to the mentor. Having been measured by the mentor method/measuring system the adept concentration is compared with the mentor concentration and the absolute and/or the relative difference is calculated. When using graphs to monitor bias over time, it is an advantage to normalize the results using the following equation, Normalized=((AdeptMentor)/Mentor)*100. The results are thereby expressed as percentage deviation from the $100 \%$ measured by the mentor. Trends in bias over time can thereby be monitored using, for example, the Levey-Jennings plot [73,74]. Linear regression and bias plots complement the Levey-Jennings plot by showing the relation between bias and the measurement level expressed on absolute and relative scales Figure 7.

Mentor-adept methods provide ideal data for partitioning overall measurement uncertainty by calculating components of variation using a top-down approach and analysis of variance/variance component analysis as originally suggested by Maroto et al. [75,76]. The measurement method or system making the largest contribution to the overall measurement uncertainty may be identified in this manner and measures taken to minimize or eliminate the largest contributions to the overall measurement uncertainty. Several more elaborate calculation methods have been developed for this purpose [33,49,55,77-90].

There are, however, at least two important obstacles to the practice of patient-sample facilitated secondary adjustment within a laboratory organization. 1) If the end-user-laboratories do their own secondary adjustment using fresh natural patient samples in order to minimize bias between measurement methods and systems from different manufacturers, it makes it difficult for the producers to shoulder their full responsibility in relation to the authorities, including the $\mathrm{EU}$ [15] and the US FDA. Secondary adjustment may, therefore, challenge and possibly jeopardize certified measurement systems. Furthermore, each company will then lose an important tool for detecting bias between measurement method and systems of their own mak- ing and corresponding measurement methods and systems located elsewhere. 2) Organizations or companies organizing proficiency testing programs will find difficulties in grouping measurements methods in producer- and method-oriented categories if the users of the measurement methods perform patient-sample facilitated secondary adjustment.

\section{A mentor laboratory and its consequences}

The bias of the mentor laboratory itself for a certain measurand should be judged by the principles applied for accreditation and proficiency testing. The laboratories currently applying the mentor-adept principles are usually accredited according to ISO 17025 or ISO 15189. The principle for bias-minimization has been accepted by the accreditation authorities. There is no other particular certification, and hardly any need for one, given accreditation and regular external inspections.

Among the consequences of implementing mentor-adept quality control principles in a conglomerate of laboratories, is that since the adept laboratories are regularly controlled by commutable control materials (fresh patient samples), the adept laboratories may not need to participate in external quality control/proficiency testing schemes since their mentor laboratory participates. This may reduce costs but risks isolating the adept laboratories from the community of laboratories participating in regular external quality control/proficiency testing schemes.

\section{Bias in total error \& uncertainty approaches}

Two different perspectives are commonly applied when describing measurement methods: focusing on the 'total error' (measure of a combination of random and systematic error); and focusing on the uncertainty of the results obtained by the measurement methods. For the sake of convenience, we will call the former 'total error approach' and the latter 'uncertainty approach'. The total error approach has been widely adopted in English speaking countries and in Germany in a special form (RiLi-BAEK) (see below).

With time the total error and uncertainty approaches have converged, as aptly explained by Rozet at al. [91].

\section{Uncertainty approach}

Measurement uncertainty encloses the interval of measurement results within which the true value of the measured quantity lies with a given probability. In contrast to total error methods approaches, uncertainty is primarily important for the users of the measurement results and is amongst the main determinants of its fitness for a particular purpose, for example, in healthcare. 


$$
U=k * u_{c}
$$

Where $\mathrm{U}=$ expanded measurement uncertainty; $\mathrm{u}_{\mathrm{c}}=$ combined standard uncertainty; $k=$ coverage factor.

The principles of general metrology, including the principles of expressing measurement uncertainty $[4,82]$, are increasingly being adopted in clinical chemistry around the world. The uncertainty methods regard the properties of a measurement method or a group of measurement methods in the perspective of the users of the measurement results rather than in the perspective of the laboratory. The expression of uncertainty in clinical chemistry aims to aid the user in making informed decisions on whether a treatment has had or is having sufficient quantitative effects.

GUM [4] defines the concepts, terms and practical performance of the calculations of measurement uncertainties. It unifies the many approaches earlier used in different fields of metrology for expressing measurement uncertainty.

\section{Total error approach}

According to its original definition, one-sided total error (TE) is the absolute value of the bias plus sample estimate of two standard deviations $[5,92]$ :

$$
T E=\mid \text { Bias } \mid+2 * s
$$

It has later [93] been broadened to the following more general expression:

$$
T E=\mid \text { Bias } \mid+Z * s
$$

where $s$ is the sample standard deviation observed during validation or verification studies $\mathrm{Z}$ may be decided to be between two and six depending on the purpose. "Most commonly, a Z-value of two is used in the reports from peer comparison programs, whereas in method validation studies, multiple values can be considered" [93]. total error serves as measurement quality requirement for single measurement methods and sets an upper limit of the interval of the combination of the imprecision and bias tolerable in a single measurement.

The total error approach in its original form directly adds standard deviation and the bias (Figure 8) to get the total error whereas the established principles of uncertainty calculation in metrology add the squares of the imprecision and take the square root of the sum of squares according to the pythagorean theorem.

A further broadening of the concept of total error toward the uncertainty concept is its use in proficiency
(A)

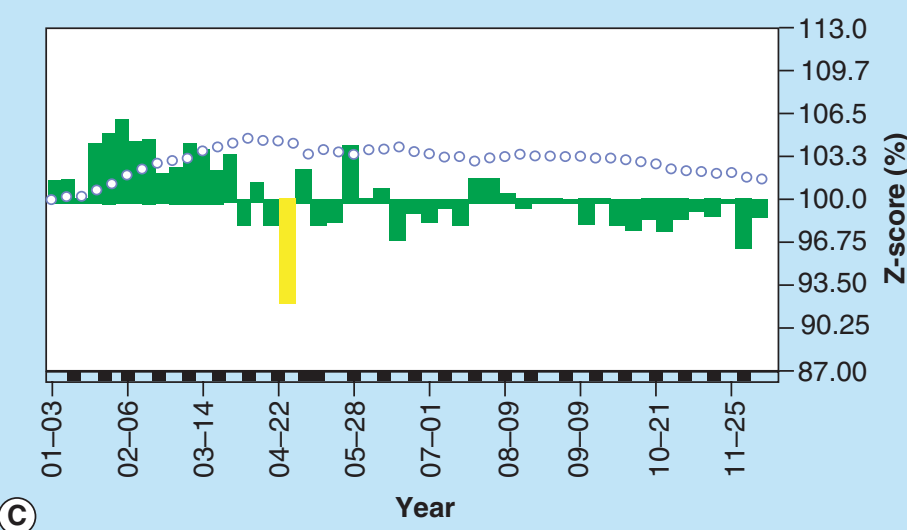

(C)

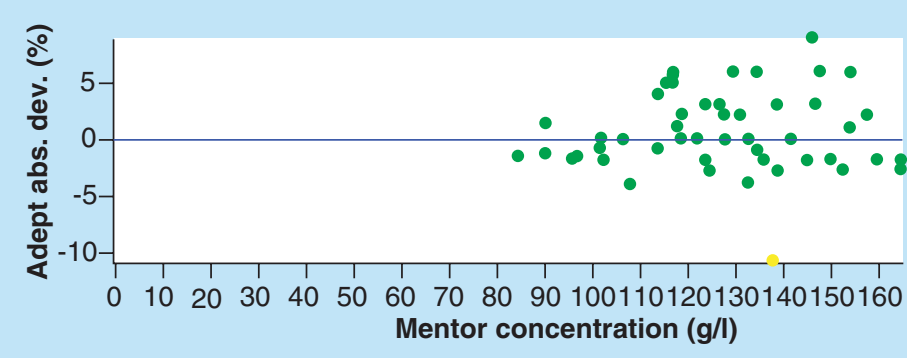

(B)

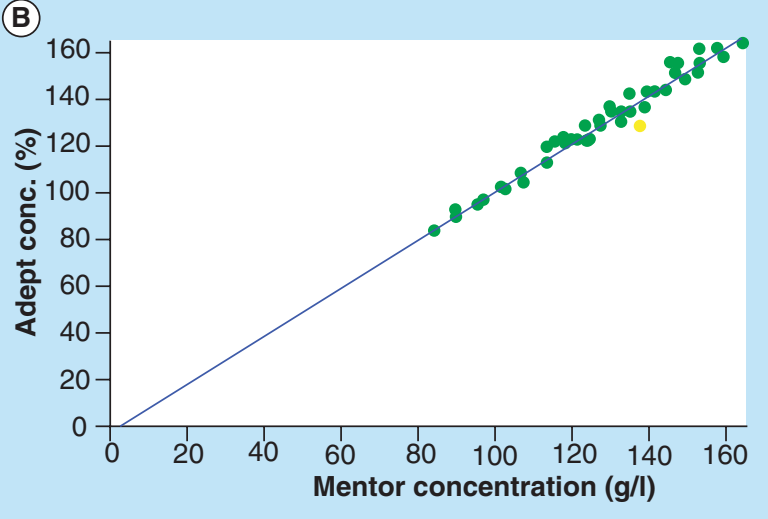

(D)

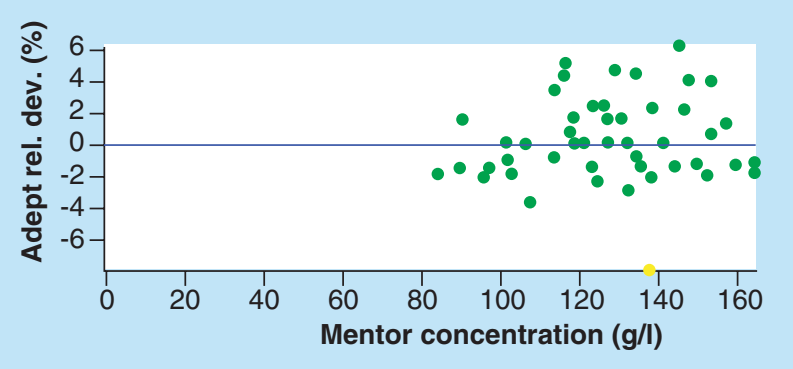

Figure 7. A mentor (split sample) technique for quality control. The results of an adept laboratory are compared with a mentor laboratory when measuring the concentration of hemoglobin in whole blood. (A) A Levy-Jennings plot of the mentor results around the optimum of $100 \%$. Each horizontal line represents one standard deviation. (B) A linear regression of the mentor concentrations (x-axis) compared with the adept concentrations (y-axis). (C \& D) Bias plots of the absolute and relative deviation of the adept results Reproduced from [46]. 
testing programs to calculate the total error on the basis of the intermediate precision and the bias observed on internal quality control materials or materials for proficiency testing programs [93]. The shortest observation period is commonly at least 6 months using the lab's mean versus some overall mean for a method subgroup or the mean from the total peer group.

The total error is intended to be predictive of the variation expected in the test results used in diagnosing diseases and monitoring treatment results. Used in this way the 'total error' calculations are similar to the 'bottom up' calculation of measurement uncertainty with one crucial difference: measurement uncertainty methods demand that known bias is eliminated whereas total error approaches incorporate bias. Incorporating bias in the calculation of the total error is also inherent in the approaches described by Krouwer [94-98]. The CLSI standard EP21-A, of which Krouwer is the main author [99], presents a well-developed view of total error and a number of alternative methods for calculating it. As described, it is quite close to the uncertainty concept.

In 1974, when Westgard et al. [5] introduced the concept of total error, the purpose was to present a quantitative method 'to judge whether an analytical method has acceptable precision and accuracy'. The authors recommended that the acceptability of the performance of a method should be judged by comparing
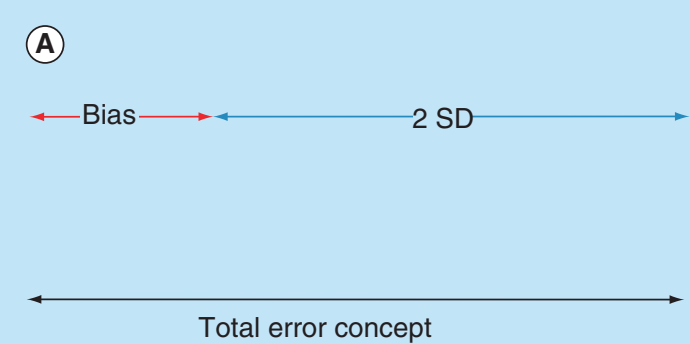

(B)

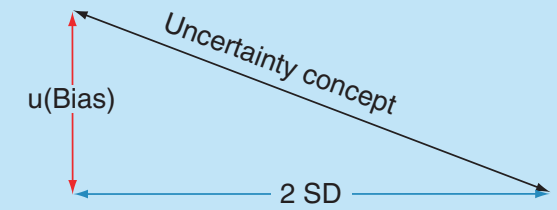

Figure 8. Combining components of uncertainty.

(A) The total error concept where the components are added linearly, (B) the uncertainty concept including RiLi-BAEK where the components are added as variances (squared components) as in the pythagorean theorem. The bias for the total error concept is the measured bias and for the uncertainty concept is the $\mathrm{u}$ (bias) which is any uncertainty of the bias component including. its observed total error to the size of a defined allowable total error. This was a response to the then common practice in laboratories to consider imprecision and bias as components of error whose acceptability sometimes were evaluated separately. When the laboratories decreased or even stopped making replicate measurements altogether, it was important to focus on the importance of the bias in the acceptability of the method itself Figure 9.

Evidently total error $=\mid$ Bias $\mid+2{ }^{*}$ is larger than $\mid$ Bias $\mid+1.65^{*}$ shich represents the absolute value of the bias and one-sided estimate of the $95 \%$ confidence limit for the random error. The US FDA has adopted the total error name and modified it to mean total error $=\mid$ Bias $\mid+1.65{ }^{*}$ s [9] thus bringing it one step nearer to the international concepts of uncertainty and GUM. This has also been accepted by Westgard et al., the originators of the concept [92].

If the bias for a measurement method or system is known, it is difficult to see the logic in including it in the calculation of the total error rather than eliminating it by re-calibration. If the bias cannot be determined, it is unknown and cannot be eliminated.

Another complication of adding bias is that the bias is a scalar whereas the imprecision is an expression of a probability distribution of random errors. They are of two different dimensions and adding them in a total error means losing the possibility of using total error for estimating the uncertainty of individual results.

The total error approach, as originally conceived, does not focus on describing a confidence interval or giving solid technical guidance on the clinical acceptability of individual methods. Total error of methods can be used to compare the performance of methods and measuring systems and provide a ranking tool for inter-laboratory comparison or comparison with analytical goals calculated and expressed in the same way. Total error methods address the question "how should the laboratory define the quality goal?" [92].

Laboratories increasingly focus on the perspectives and needs of the end users (the patients and healthcare personnel), uncertainty perspectives applied in medical practice gain increased relevance. A particular feature of the uncertainty concept is that it can be used to describe both the performance of a measuring system and the single, individual value.

\section{RiLi-BAEK (Richtlinien der Bundesärztekammer)} RiLi-BAEK are the Guidelines ("Rili") of the German Federal Medical Council (Bundesärztekammer), now available in the 2013 version [6]. Similar to the CLIA limits in the US, they set minimum requirements for the quality of quantitative test results in medical laboratories. The approach and principles used by RiLi- 


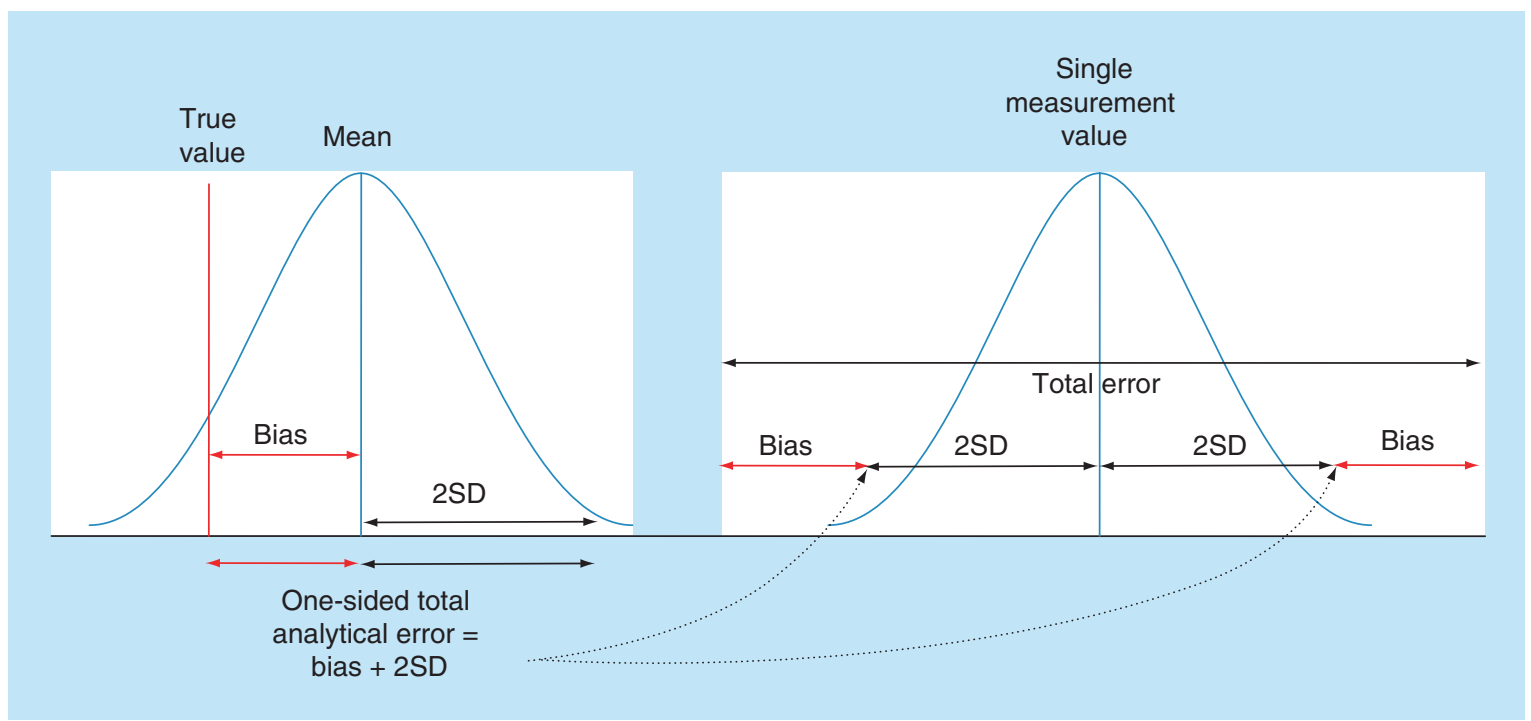

Figure 9. The total error (TE) = total error of a measurement method as originally defined by Westgard et al. [5].

BAEK are very similar to the total error concepts, although the methods for calculating the total error differ somewhat $[6,100]$. The RiLi-BAEK guidelines that govern medical devices in laboratory medicine in Germany are tied to the European IVD directive and the ISO standards for example, 15189. Therefore, they are not only a list of upper limits for total measurement error but stipulate an approach for quality control, quality improvements and accreditation of laboratories. In the view of the present authors it would be more appropriate to base the criteria primarily on the fitness of purpose for patients and healthcare workers.

$$
\Delta_{\max }=\sqrt{k^{2} * s^{2}+B i a s^{2}}
$$

Where $\Delta_{\max }=$ maximum allowable error when measuring a control sample; $s=$ standard deviation; $\mathrm{k}=\mathrm{a}$ statistical coverage factor that depends on the purpose; bias = mean concentration measured in the control samples - target value of the control sample provided by its manufacturer.

RiLi-BAEK appears to have inherited its technical focus on the upper limit of measurement uncertainty from the initial total error approach $[5,92]$ and from the US FDA [9]. At the same time, it has embraced the principles for error calculation and uncertainty estimation from the GUM [4] and other authorities in metrology.

Total error and similar approaches reporting and setting limits are evidently suitable for situations where a single measurement method is used for monitoring the concentrations of a measurand over time in a patient, for example, a measurement method for selfmonitoring of glucose. However, when samples from the same patient during extended periods of monitoring are measured using several different measurement methods and systems in different laboratories, bias between measurement methods becomes increasingly important for the uncertainty affecting the clinical decisions. In this case, attempts to eliminate bias become more crucial and more advanced models for calculating uncertainty are needed $[46,101]$.

\section{Conclusion}

Uncertainty of the high-volume measurement methods in clinical chemistry has decreased substantially with the advent of highly automated measurement methods and reference measurement systems during the last five decades. The effect of the repeatability and day-to-day variation on the overall uncertainty has decreased substantially during this time period, but bias to a less degree.

Reference measurement methods and commutable reference materials constitute the foundations of all efforts for eliminating bias and reducing measurement uncertainty in clinical chemistry. Internationally the bias has been addressed by several important organizations and projects, including JCTLM and The American association of clinical chemistry. Despite their widespread use, important challenges remain for the end users due to remaining bias between measurement methods and systems from different and or the same manufacturers. This is particularly evident for immunochemical methods specific for different epitopes of the molecules measured.

In clinical chemistry the end users have an abundant supply of the optimal commutable material useful for minimizing bias - fresh patient samples. Split-sample/mentor-adept methods combined with secondary adjustments are optimal for reducing any remaining clinically important bias in particular in 
conglomerates of laboratories measuring samples from the same patient.

\section{Future perspective}

Further developments in reference measurement systems are likely continue to play the major role in minimizing bias in clinical chemistry during in the next two decades. Reference measurement systems are, however, unlikely to solve all the most complex bias issues, for example, in the fields of immunochemistry. Natural patient samples are commutable and in abundant supply in the laboratories of clinical chemistry. They represent an asset that is already available and is likely to be increasingly used for minimizing bias still further using split-sample/mentor-adept techniques.

Financial \& competing interests disclosure

Financial support for this work was provided by the County Council of Östergötland and from Linköping University. The authors have no other relevant affiliations or financial involvement with any organization or entity with a financial interest in or financial conflict with the subject matter or materials discussed in the manuscript apart from those disclosed.

No writing assistance was utilized in the production of this manuscript.

\section{Executive summary}

- The concepts of trueness expressed as bias and accuracy expressed as measurement uncertainty have been agreed on by authoritative international organizations.

- Their proper meaning and nomenclature needs to be implemented globally.

- Bias in clinical chemistry is a clinically important challenge.

- Bias in clinical chemistry has been and is being decreased by reference measurement systems created and maintained by several organizations and by manufacturers of measurement methods and systems.

- Clinically important bias, however, remains and represents a particular challenge when diagnosing and monitoring disease where minute changes in concentrations have major clinical consequences for example, in diabetes mellitus.

- Bias can be measured and monitored.

- Bias between measurement systems and methods may be due matrix effects.

- Natural patient samples are fully commutable and, therefore, optimal for estimating and eliminating bias using natural patient samples in split-sample/mentor-adept methods.

- Variable bias components become random errors over time and can be treated by techniques of variance component analysis.

- The longer time period observed the random error increases and the bias decreases since some bias components become random over time.

- Bias can and should be eliminated.

- There is current reluctance in using secondary adjustment of measured concentrations using mentor-adept methods due to regulatory issues including the EU in vitro directive.

\section{References}

Papers of special note have been highlighted as:

- of interest; $\bullet$ of considerable interest

1 Mcnaught AD, Wilkinson A. International Union of Pure and Applied Chemistry: Compendium of chemical terminology: IUPAC recommendations. (2nd Edition). Blackwell Science, Oxford, UK (1997).

2 EP09-A3 Measurement Procedure Comparison and Bias Estimation Using Patient Samples; Approved Guideline (3rd Edition). Clinical and Laboratory Standards Institute, PA, USA (2013).

- Important guideline for bias estimation using commutable samples = patient samples. However, it does not cover secondary adjustment of concentrations.

3 Kallner A, Khorovskaya L, Pettersson T. A method to estimate the uncertainty of measurements in a conglomerate of instruments/laboratories. Scand. J. Clin. Lab. Invest. 65(7), 551-558 (2005).

4 JCGM. Evaluation of measurement data - guide to the expression of uncertainty in measurement (2008). www.bipm.org/utils/common/documents/jcgm/ JCGM_100_2008_E.pdf.

- A general guide to the expression of measurement uncertainty. It deals mainly with 'bottom up' approaches but also makes clear that 'top down' approaches most commonly used in chemistry are appropriate and that bias should be eliminated.

5 Westgard JO, Carey RN, Wold S. Criteria for judging precision and accuracy in method development and evaluation. Clin. Chem. 20(7), 825-833 (1974).

6 Richtlinie der Bundesärztekammer zur Qualitätssicherung laboratoriumsmedizinischer Untersuchungen. Bundesärztekammer, Berlin, Germany (2013).

7 Barwick V, Prichard E. Terminology in Analytical Measurement - Introduction to VIM 3. (2011). www.eurachem.org

8 JCGM. International vocabulary of metrology - Basic and general concepts and associated terms ( $3^{\text {rd }}$ Edition). (2012). www.bipm.org/utils/common/documents/jcgm/ JCGM_200_2012.pdf 
-. The definitive guide to concepts and terms in all fields of metrology.

9 Recommendations for clinical laboratory improvement amendments of 1988 (CLIA) waiver applications for manufacturers of in vitro diagnostic devices. US Food and Drug Administration, MD, USA (2008).

10 Greenberg N. Update on current concepts and meanings in laboratory medicine - Standardization, traceability and harmonization. Clin. Chim. Acta 432, 49-54 (2014).

11 Menditto A, Patriarca M, Magnusson B. Understanding the meaning of accuracy, trueness and precision. Accred. Qual. Assur. 12, 45-47 (2007).

12 Thompson M. Towards a unified model of errors in analytical measurement. Analyst 125, 2020-2025 (2000).

13 Iso 17511:2003 In vitro Diagnostic Medical Devices Measurement OfQuantities In Biological Samples - Metrological Traceability Of Values Assigned To Calibrators And Control Materials. International Organization for Standardization, Geneva, Switzerland (2003).

14 Armbruster D, Miller RR. JCTLM: A global approach to promote the standardisation of clinical laboratory test results. Clin. Biochem. Rev. 28(3), 105-113 (2007).

15 EU. Directive 98/79/EC of the European Parliament and of the Council of 27 October 1998 on in vitro diagnostic medical devices. (1998).

http://eur-lex.europa.eu/LexUriServ/LexUriServ. do?uri=CELEX:31998L0079:EN:NOT.

16 Panteghini M. Traceability as a unique tool to improve standardization in laboratory medicine. Clin. Biochem. 42(4-5), 236-240 (2009).

17 Infusino I, Frusciante E, Ferrero CA, Panteghini M. Commutability of two JCTLM-listed secondary reference materials for two commercial lithium assays. Clin. Chim. Acta 414, 152-153 (2012).

18 Siekmann L. Establishing measurement traceability in clinical chemistry. Accred. Qual. Assur. 9(1-2), 5-17 (2004).

19 Siekmann L. Implementation of the concept of traceability in laboratory medicine in external quality assessment. Clin. Biochem. 42(4-5), 293-294 (2009).

20 Siekmann L. Metrological traceability - a concept for standardization in laboratory medicine. Clin. Chem. Lab. Med. 51(5), 953-957 (2013).

21 Panteghini M. Application of traceability concepts to analytical quality control may reconcile total error with uncertainty of measurement. Clin. Chem. Lab. Med. 48(1), 7-10 (2010).

22 Tate JR, Johnson R, Barth J, Panteghini M. Harmonization of laboratory testing - Current achievements and future strategies. Clin. Chim. Acta 432, 4-7 (2013).

23 Müller MM. Tracebility in laboratory medicine. Accred. Qual. Assur. 8, 340-345 (2003).

24 Miller WG, Myers GL, Lou Gantzer M et al. Roadmap for harmonization of clinical laboratory measurement procedures. Clin. Chem. 57(8), 1108-1117 (2011).

25 Miller WG, Tate JR, Barth JH, Jones GR. Harmonization: The Sample, the Measurement, and the Report. Ann. Lab. Med. 34(3), 187-197 (2014).
26 Weykamp C, Eckfeldt J, Vesper H et al. Toolbox of technical procedures to be considered when developing a process to achieve harmonization for a measurand. (2013). www.harmonization.net/Resource/Documents/Tool_ Box_2013.pdf

27 Bonini P, Plebani M, Ceriotti F, Rubboli F. Errors in laboratory medicine. Clin. Chem. 48(5), 691-698 (2002).

28 Misra S, Barth JH. How good is the evidence base for test selection in clinical guidelines? Clin. Chim. Acta 432, 27-32 (2014).

29 Simundic AM, Cornes M, Grankvist K et al. Survey of national guidelines, education and training on phlebotomy in 28 European countries: An original report by the European Federation of Clinical Chemistry and Laboratory Medicine (EFLM) working group for the preanalytical phase (WGPA). Clin. Chem. Lab. Med. 51(8), 1585-1593 (2013).

30 Aakre KM, Langlois MR, Watine J et al. Critical review of laboratory investigations in clinical practice guidelines: Proposals for the description of investigation. Clin. Chem. Lab. Med. 51(6), 1217-1226 (2013).

31 Collinson PO, Van Dieijen-Visser MP, Pulkki K et al. Evidence-based laboratory medicine: how well do laboratories follow recommendations and guidelines? The Cardiac Marker Guideline Uptake in Europe (CARMAGUE) study. Clin. Chem. 58(1), 305-306 (2012).

32 Plebani M. Harmonization in laboratory medicine: the complete picture. Clin. Chem. Lab. Med. 51(4), 741-751 (2013).

33 Alvarez-Prieto M, Jiménez-Chacón J, Montero-Curbelo Á. Do we need to consider metrological meanings of different measurement uncertainty estimations? Accred. Qual. Assur. 14, 623-634 (2009).

34 Thienpont LM. Accuracy in clinical chemistry - Who will kiss Sleeping Beauty awake? Clin. Chem. Lab. Med. 46(9), 1220-1222 (2008).

35 Rao CR, Kleffe J. Estimation of Variance Components and Applications. Elsevier Science Publications, Amsterdam, Netherlands (1988).

36 Cox DR, Solomon PJ. Components of Variance. CRC Press Inc., FL, USA (2003).

37 Searle SR, Casella G, Mccullogh CE. Variance Components. John Wiley \& Sons, Inc., NY, USA (1992).

38 Galen RS, Gambino SR. Beyond Normality: The Predictive Value and Efficiency of Medical Diagnoses. John Wiley \& Sons, Inc., NY, USA (1975).

39 Knottnerus JA, Buntinx F. The Evidence Base of Clinical Diagnosis: Theory and Methods of Diagnostic Research (2nd Edition). Blackwell Publishing Ltd, Oxford, UK (2008).

40 Krzanowski WJ, Hand DJ. ROC Curves for Continuous Data. CRC Press Inc., FL, USA (2009).

41 Ricós C, Alvarez V, Cava F et al. Current databases on biological variation: pros, cons and progress. Scand. J. Clin. Lab. Invest. 59(7), 491 - 500 (1999).

42 Fraser CG. Interpretation of Clinical Chemistry Laboratory Data. Blackwell Scientific, Oxford, UK (1986).

43 Ricos C, Alvarez V, Cava F et al. desirable specifications for total error, imprecision, and bias, derived from intra- and 
inter-individual biologic variation. (2014) www.westgard.com/biodatabase1.htm.

44 Petersen PH, Jorgensen LG, Brandslund I, De Fine Olivarius $\mathrm{N}$, Stahl M. Consequences of bias and imprecision in measurements of glucose and hbalc for the diagnosis and prognosis of diabetes mellitus. Scand. J. Clin. Lab. Invest. Suppl. 240, 51-60 (2005).

45 Petersen PH, de Verdier CH, Groth T, Fraser CG, Blaabjerg $\mathrm{O}$, Horder M. The influence of analytical bias on diagnostic misclassifications. Clin. Chim. Acta 260(2), 189-206 (1997).

46 Theodorsson E. Validation and verification of measurement methods in clinical chemistry. Bioanalysis 4(3), 305-320 (2012).

47 Kateman G, Buydens L. Quality Control in Analytical Chemistry. John Wiley \& Sons, NY, USA (1993).

48 Montgomery DC. Introduction to statistical quality control (6th Edition). Wiley, Hoboken, NJ, USA (2009).

49 Ellison SLR, Farrant TJ, Barwick V. Royal Society of Chemistry (Great Britain): Practical statistics for the analytical scientist : a bench guide (2 $2^{\text {nd }}$ Edition). RSC Publishing, Cambridge, UK (2009).

- A valuable overview of statistical methods used in laboratories.

50 Becker D, Christensen R, Currie L et al. Use of NIST standard reference materials for decisions on performance of analytical chemical methods and laboratories. (1992). www.nist.gov/mml/csd/inorganic/upload/NIST_ SpecialPub829.pdf

51 Natrella MG. Experimental Statistics. John Wiley \& Sons, NY, USA (1984).

52 Kallner A. Laboratory Statistics: Handbook of Formulas and Terms (1st Edition). Elsevier, Amsterdam, Netherlands (2013)

- A valuable overview of statistical methods used in clinical laboratories.

53 Castrup H. Estimating bias uncertainty. (2001). www. isgmax.com/articles_papers/bias\%20uncertainty.pdf

54 Devenathan S, Rollins DK, Vardeman SB. A new approach for improved identification of measurement bias. Comput. Chem. Eng. 24, 2755-2764 (2000).

55 Magnusson B, Ellison SL. Treatment of uncorrected measurement bias in uncertainty estimation for chemical measurements. Anal. Bioanal. Chem. 390 (1), 201-213 (2008).

56 Magnusson B, Leito I. Personal communication, draft leaflet from EuraChem. Measurement uncertainty and traceability working group. www.eurachem.org. (2014).

57 Dietzen DJ, Queen SF, Solomon SS. Matrix-dependent bias in total thyroxine measurement on the Beckman Access. Clin. Chim. Acta 316(1-2), 171-174 (2002).

58 Sobas F, Benattar N, Bellisario A et al. Impact of quality control matrix effect: Application to the calculation of uncertainty of measurement in one-stage clotting factor VIII assay. Blood Coagul. Fibrinolysis 21(5), 498-501 (2010).

59 Unsal I, Coskun A, Serteser M, Inal TC, Ozpinar A. Toward standardization of quality assessment in laboratory medicine by using the same matrix samples for both internal and external quality assessments. Accred. Qual. Assur. 15, 621-627 (2010).

60 Zegers I, Beetham R, Keller T et al. The importance of commutability of reference materials used as calibrators: the example of ceruloplasmin. Clin. Chem. 59(9), 1322-1329 (2013).

61 Miller WG, Myers GL. Commutability still matters. Clin. Chem. 59(9), 1291-1293 (2013).

62 White GH. Metrological traceability in clinical biochemistry. Ann. Clin. Biochem. 48 (Pt 5), 393-409 (2011).

63 Vesper HW, Miller WG, Myers GL. Reference materials and commutability. Clin. Biochem. Rev. 28(4), 139-147 (2007).

64 Miller WG, Myers GL, Rej R. Why commutability matters. Clin. Chem. 52(4), 553-554 (2006).

65 Franzini C, Ceriotti F. Impact of reference materials on accuracy in clinical chemistry. Clin. Biochem. 31(6), 449-457 (1998).

66 Franzini C. Commutability of reference materials in clinical chemistry. J. Int. Fed. Clin. Chem. 5(4), 169-173 (1993).

67 Vesper HW, Thienpont LM. Traceability in laboratory medicine. Clin. Chem. 55(6), 1067-1075 (2009).

68 Thienpont LM, Van Nuwenborg JE, Stockl D. Intrinsic and routine quality of serum total potassium measurement as investigated by split-sample measurement with an ion chromatography candidate reference method. Clin. Chem. 44(4), 849-857 (1998).

69 Solvik UO, Stavelin A, Christensen NG, Sandberg S. External quality assessment of prothrombin time: the splitsample model compared with external quality assessment with commercial control material. Scand. J. Clin. Lab. Invest. 66(4), 337-349 (2006).

70 Stavelin A, Petersen PH, Solvik UO, Sandberg S. External quality assessment of point-of-care methods: model for combined assessment of method bias and single-participant performance by the use of native patient samples and noncommutable control materials. Clin. Chem. 59(2), 363-371 (2013).

71 Dybkaer R. From total allowable error via metrological traceability to uncertainty of measurement of the unbiased result. Accred. Qual. Assur. 4(9-10), 401-405 (1999).

- A classic paper written by one of the nesters of metrology. It makes a solid case for the elimination of bias and secondary adjustment of concentrations.

72 Kuttatharmmakul S, Massart DL, Smeyers-Verbeke J. Comparison of alternative measurement methods: Determination of the minimal number of measurements required for the evaluation of the bias by means of interval hypothesis testing. Chemometr. Intell. Lab. 52(1), 61-73 (2000).

73 Levey S, Jennings ER. The use of control charts in the clinical laboratory. Am. J. Clin. Pathol. 20, 1059-1066 (1950).

74 Henry RJ, Seaglove M. The running of standards in clinical chemistry and the use of the control chart. J. Clin. Pathol. 5, 305-311 (1952). 
75 Maroto A, Riu J, Boque R, Rius FX. Estimating uncertainties of analytical results using information from the validation process. Anal. Chim. Acta 391(2), 173-185 (1999).

76 Committee AM. Uncertainty of measurement: Implications of its use in analytical science. Analyst 120, 2303-2305 (1995).

77 Barwick VJ, Ellison SLR. Estimating measurement uncertainty using a cause and effect and reconciliation approach Part 2. Measurement uncertainty estimates compared with collaborative trial expectation. Anal. Commun. 35(11), 377-383 (1998).

78 Ellison SL. ISO uncertainty and collaborative trial data. Accred. Qual. Assur. 3, 95-100 (1998).

79 Ellison SLR, Barwick VJ. Estimating measurement uncertainty: Reconciliation using a cause and effect approach. Accred. Qual. Assur. 3(3), 101-105 (1998).

Barwick VJ, Ellison SL, Rafferty MJQ, Gill RS. The evaluation of measurement uncertainty from method validation studies Part 2: The practical application of a laboratory protocol. Accred. Qual. Assur. 5, 104-113 (2000).

81 Ellison SLR, Williams A. Eurachem/CITAC Guide Quantifying Uncertainty in Analytical Measurement (3rd Edition). (2012). www.eurachem.org/index.php/publications/guides/quam Dell GE, Hibbert DB. Reply to "comment": Do we really need to account for run bias when producing analytical results with stated uncertainty? Analyst 132(12), 1272-1274 (2005).

83 O'donnell GE, Hibbert DB. Treatment of bias in estimating measurement uncertainty. Analyst 130(5), 721-729 (2005).

84 Hibbert DB. Systematic errors in analytical measurement results. J. Chromatogr. A 1158(1-2), 25-32 (2007).

85 Kadis R. Do we really need to account for run bias when producing analytical results with stated uncertainty? Comment on 'Treatment of bias in estimating measurement uncertainty' by G. E. O'Donnell and D. B. Hibbert. Analyst 132(12), 1272-1274; discussion 1275-1277 (2007).

86 Synek V. Attempts to include uncorrected bias in the measurement uncertainty. Talanta 65(4), 829-837 (2005).

Phillips SD, Eberhardt KR. Guidelines for expressing the uncertainty of measurement results containing uncorrected bias. J. Res. Natl Inst. Stand. Technol. 102(5), 577-585 (1997).
88 Hasselbarth W. Accounting for bias in measurement uncertainty estimation. Accred. Qual. Assur. 9, 509-514 (2004).

89 Hässelbarth W. Measurement uncertainty procedures revisited: Direct determination of uncertainty and bias handling. Accred. Qual. Assur. 3, 418-422 (1998).

90 Rozet E, Marini RD, Ziemons E et al. Total error and uncertainty: Friends or foes? Trends Anal. Chem. 30(5), 797-806 (2011).

91 Westgard JO, Westgard SA. Total analytic error. From concept to application. (2013)www.aacc.org/publications/ cln/2013/september/Pages/Total-Analytic-Error.aspx\#

92 Westgard JO. The meaning and application of total error. (2007).

www.westgard.com/essay111.htm?format=phocapdf

93 Krouwer JS. Multi-factor designs IV. How multi-factor designs improve the estimate of total error by accounting for protocol-specific biases. Clin. Chem. 37, 26-29 (1991).

94 Krouwer JS. Estimating total analytical error and its sources. Techniques to improve method evaluation. Arch. Pathol. Lab. Med. 116(7), 726-731 (1992).

95 Krouwer JS. How to improve total error modeling by accounting for error sources beyond imprecision and bias. Accred. Qual. Assur. 47(7), 1329-1330 (2001).

96 Krouwer JS. Setting performance goals and evaluating total analytical error for diagnostic assays. Clin. Chem. 48(6 Pt 1), 919-927 (2002).

97 Krouwer JS. Simulating total error while excluding results underestimates total error. Clin. Chem. 56(9), 1505-1506; author reply 1506 (2010).

98 Krouwer JS, Astles JR, Cooper WG et al. Estimation of Total Analytical Error for Clinical Laboratory Methods; Approved Guideline, EP21-A. Clinical and Laboratory Standards Institute, PA, USA (2003).

99 Bauersfeld W. RiliBAK-a new computation concept. Clin. Lab. 52(11-12), 689-694 (2006).

100 Phillipov G, Phillips PJ. Components of total measurement error for hemoglobin A(1c) determination. Clin. Chem. 47(10), 1851-1853 (2001). 